\title{
BMJ Open Effectiveness of a digital dietary intervention program targeting young adults before parenthood: protocol for the PREPARED randomised controlled trial
}

Nina Cecilie Øverby (D), Anine C Medin, Erlend Larsen Valen, Lorentz Salvesen, Andrew Keith Wills, Dagrun Engeset, Frøydis N Vik, Elisabet R Hillesund

To cite: Øverby NC, Medin AC, Valen EL, et al. Effectiveness of a digital dietary intervention program targeting young adults before parenthood: protocol for the PREPARED randomised controlled trial. BMJ Open 2021;11:e055116. doi:10.1136/ bmjopen-2021-055116

- Prepublication history for this paper is available online. To view these files, please visit the journal online (http://dx.doi. org/10.1136/bmjopen-2021055116).

Received 02 July 2021 Accepted 04 November 2021

Check for updates

(c) Author(s) (or their employer(s)) 2021. Re-use permitted under CC BY-NC. No commercial re-use. See rights and permissions. Published by BMJ.

Department of Nutrition and Public Health, Universitetet $i$ Agder, Kristiansand, Vest-Agder, Norway

Correspondence to Professor Nina Cecilie Øverby; nina.c.overby@uia.no

\section{ABSTRACT}

Introduction The importance of preconception health for lifelong physical and mental health in the next generation has gained increasing recognition in recent years. Preconception paternal and maternal risk factors such as obesity and inadequate diet affect the metabolic and cardiovascular health of their offspring later in life. This highlights the importance of diet and dietary behaviour in the years before parenthood. In our project, PREPARED, we will evaluate the effectiveness of a digital intervention targeting young adults. Our primary aim is to improve participants' preconception diet, and our secondary aim is to improve preconception quality of life and maternal and child perinatal outcomes.

Methods and analysis We plan to recruit 7000 men and women individually, aged 20-35 years without children, to be randomised to an intervention or a control group. The intervention group will receive access to a digital resource for 6 months promoting a healthy diet for their health now, later in life and for the next generation. Follow-up is up to 20 years or until they have their first child. To evaluate intervention effects, we will collect dietary data $(2 \times 24$ hour dietary recalls and a screener). For those participants for which birth ensues, we will link study data with data from the Medical Birth Registry of Norway on maternal and child perinatal outcomes.

Ethics and dissemination The study is approved by the Regional Ethics Committee, the Norwegian Data Protection Service and our Faculty Ethical Committee (REC: 78104, NSD: 907212, FEC 20/10119). Participation is voluntary and all participants will provide informed consent. Participants can withdraw their consent without giving any reason. Findings will be communicated to the public through a project website and social media, and to professionals through conferences and peer-reviewed papers.

Trial registration number ISRCTN44294662.

\section{INTRODUCTION}

The importance of preconception health for lifelong physical and mental health in the next generation has gained increasing recognition

\section{Strengths and limitations of this study}

- This is one of the first studies to evaluate the effects of a digital dietary intervention carried out during the preconception years using a randomised controlled design.

- Pregnancy and child outcomes will be evaluated by linkage to the unique Medical Birth Registry data of Norway.

- If the intervention proves to be effective, it can be implemented at scale targeting young people to improve their current health and health for the next generation.

- Challenges relate to recruitment and retention which could compromise statistical power and validity.

in recent years. ${ }^{1}$ Preconception paternal and maternal risk factors such as obesity, inadequate diet, undernutrition, diabetes mellitus and hyperglycaemia affect the metabolic and cardiovascular health of their offspring later in life. ${ }^{12}$ In 2018, a Lancet series on preconception health reviewed the existing literature from biological, epidemiological and behavioural research and highlighted the importance of adequate nutrition in this phase. ${ }^{134}$ Combined, maternal and paternal periconceptional nutritional status provide a legacy for offspring health and development through its influence on egg and sperm integrity and thereby the very foundation for subsequent embryo development. ${ }^{356}$ The parental periconceptional nutritional status could be viewed as the biological capital acquired from a long-term diet and is a composite entity.

The preconception phase is not straightforward to define, as the exact time of conceiving is unknown, and the fertile age is wide. Stephenson et $a l^{1}$ proposed three definitions for this phase: (1) the biological 
perspective-the days to weeks before conception and embryonic development; (2) the individual perspectivestarting with a conscious intention to conceive, typically weeks to months before the pregnancy occurs; and (3) the public health perspective- the months or years from puberty with the possibility to address preconception risk factors such as diet. The preconception phase may thus range from early adolescence to late adulthood. Barker et al suggest that public health preconception initiatives should be broader and more general compared with those targeting people planning pregnancies, as many will not be motivated for health change solely by the thought of affecting a potential future child. ${ }^{4}$ There are, however, few interventions addressing this general public health perspective. ${ }^{45}$ Existing studies typically relate to improving diet in couples with compromised fertility or target women who are actively planning pregnancy. ${ }^{78}$ Research in low-income and middle-income countries settings has evaluated the effects of providing dietary supplements. ${ }^{9}$ Some studies are also in the planning, among them The Healthy Life Trajectories Initiative, the HELTI-project, which is a consortium of four studies evaluating whether an integrated intervention starting preconceptionally will reduce non-communicable disease (NCD) risk in participants' future children. ${ }^{9}{ }^{10}$ HELTI primarily recruits women (one study partner also recruits partners), and women who are planning to have children. In our study, we recruit men and women individually, regardless of partnership and plans for children. Public health actions towards the general preconception population are scarce, ${ }^{4}$ but could yield high returns at low cost and are, therefore, highly warranted and called for. ${ }^{1411}$

Poor diet is a leading risk factor for NCDs, which accounts for $80 \%-90 \%$ of the disease burden in Western countries. ${ }^{12}$ By addressing preconception diet a triple dividend is envisioned, with the potential to affect the current and future health of the targeted person as well as the health of future children. ${ }^{1}$ Aside from official recommendations on folate supplements and abstaining from alcohol while planning pregnancy, diet is hardly addressed in preconception care in Norway. National surveys suggest significant opportunities for dietary improvements in the intake of fruits, vegetables, whole grains, nuts/seeds, fish and milk as well as reduced intake of processed meat and salt among Norwegian young adults. ${ }^{13}$ In addition, our recent study of the diet of Norwegian students identified a reason for concern, showing low diet quality compared with recommendations, with a more deficient diet among men than women. ${ }^{14}$ Students constitute a large proportion of the preconception population in Norway. Hence, there is an opportunity for being better prepared for one's health and the health of the next generation. ${ }^{15}$

In our project, PREPARED, we will evaluate a newly developed digital intervention targeting young men and women aged 20-35 individually, in a randomised controlled trial. Our primary aim is to improve participants' diet and secondary aims are (1) to improve participants' quality of life and (2) improve maternal and child perinatal outcomes by following participants up to 20 years or until they have their first child.

\section{METHODS AND ANALYSIS}

This protocol paper has been written according to the Standard Protocol Items: Recommendations for Interventional Trials guidance. ${ }^{16}$

\section{Aim}

The study aims to evaluate the effectiveness of a digital dietary intervention among young adults before parenthood. We hypothesise that being exposed to the intervention will lead to improved male and female knowledge and skills concerning diet and lead to healthier preconception diet, improved health-related quality of life with potential sustained effect in future pregnancy as documented by the reduced prevalence of pregnancy complications and improved newborn anthropometry relative to the control group.

The PREPARED study is a nationwide, randomised controlled digital dietary intervention trial. Participants will be followed from inclusion until their first child is born, or a maximum of 20 years. With this long duration of data collection and yearly follow-up questionnaires regarding diet, the study provides updated information on parental diet as proximal as possible to parenthood. Data collected with this type of design can also be used to investigate diet-outcome associations.

\section{Participants}

We will recruit men and women aged 20-35 years without biological children. Inclusion criteria: participants will be eligible for the study if they are born in the years 1986-2001, have no biological children, possess an 11-digit Norwegian identification number, are literate in Norwegian/Scandinavian as the intervention content is in Norwegian only and have access to a smartphone or another digital device.

Participants will be recruited individually, regardless of whether they are in a relationship or not. As the project relates to family planning and future parenthood, one could envision that couples would like to participate together. We will inform participants in advance that only one person per couple may participate. Participants will be asked whether they are in a relationship and whether their partner participates in the study. This will be done to be able to run sensitivity analyses, omitting possible contamination of data from couples taking part in the study together and being in different intervention groups. Due to the long timeframe for this study, the current partner situation may not be the same as when participants decide to start a family. We will therefore continue to ask regularly if the partner participates in the study. We will not request the identity of the partner. 


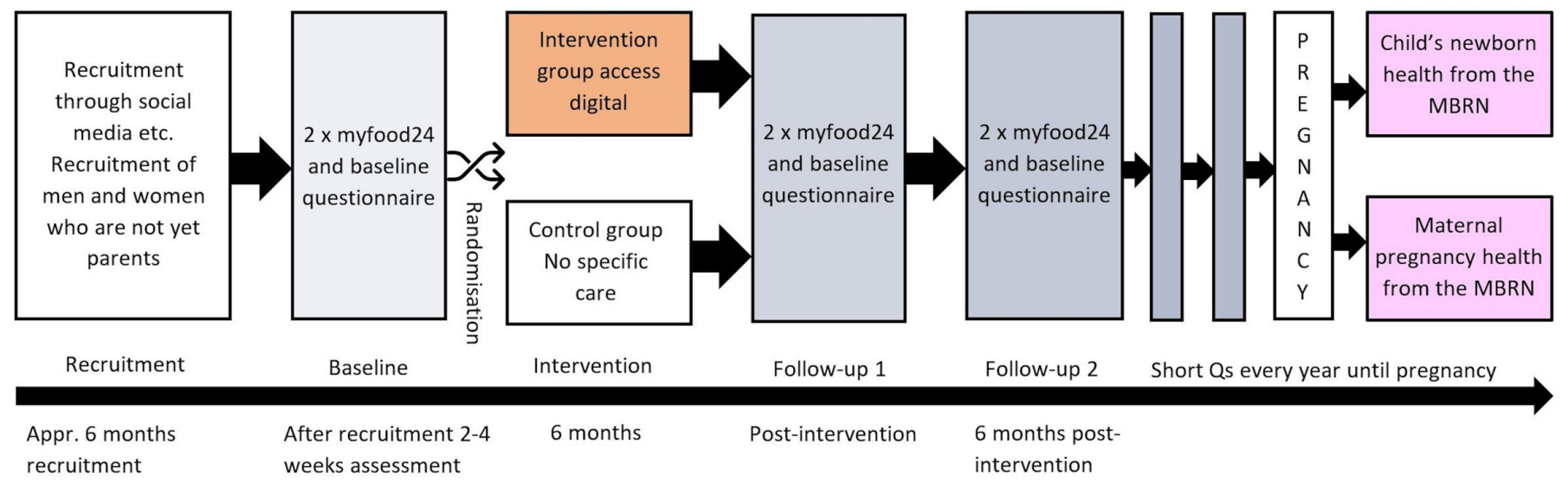

Figure 1 Timeline for the PREPARED project. MBRN, Medical Birth Registry Norway; Qs, questionnaires.

\section{Detailed study outline, recruitment and randomisation}

We will recruit among all Norwegian residents who belong to the target population and holds a Norwegian identification number. We originally planned to apply a dual recruitment strategy, targeting potential participants via both postal mail and social media. In the pilot study (see aim below), we only recruited through postal letters (August 2021). This was not a successful strategy. We will therefore recruit only through social media in the main study as we have experience of this being successful from other studies. ${ }^{17}$ The main study will start recruiting in October 2021 and continue throughout April 2022. With assistance and surveillance from the University of Agder (UiA) Communication Division, we will recruit participants through tailored social media advertisement, with text and video content adjusted according to the response. Given the use of social media recruitment, we cannot exclude recruitment of Norwegians living outside Norway. As long as they fulfil inclusion criteria they will be included. All data will be collected digitally.

An overview of the project timeline is provided in figure 1. Participants will be enrolled in the study continuously following self-registration and providing their informed consent online when logging into the baseline questionnaire.

Participants will be randomised after they have filled in a baseline questionnaire (see table 1) and completed $2 \times 24$-hour dietary recalls at least 14 days apart. Knowing

Table 1 Overview of primary (PO) and secondary (SO) outcomes and methods for the randomized controlled trial (RCT)

\begin{tabular}{|c|c|c|c|c|}
\hline Outcome & Variable & Measure & Instrument & $\begin{array}{l}\text { Sample size } \\
\text { (incl. dropout)† }\end{array}$ \\
\hline Preconception diet (PO)‡ & $\begin{array}{l}\text { Overall diet } \\
\text { Diet quality }\end{array}$ & $\begin{array}{l}\text { Food and nutrient intake } \\
\text { Diet score }\end{array}$ & $\begin{array}{l}2 \times 24 \text { hour recall (myfood } 24 \text { ) } \\
\text { Food propensity questionnaire }\end{array}$ & $211(264) \S$ \\
\hline
\end{tabular}

\begin{tabular}{|c|c|c|c|c|}
\hline \multirow[t]{3}{*}{ Pregnancy health (SO) } & Gestational weight gain (GWG) & $\begin{array}{l}\text { GWG (absolute measure and } \\
\text { relative to guidelines) }\end{array}$ & \multirow{3}{*}{$\begin{array}{l}\text { Data retrieved from MBRN as } \\
\text { recorded in pregnancy records } \\
\text { and birth certificate }\end{array}$} & 1100 (1375)ף \\
\hline & Hypertensive disorders & Preeclampsia yes/no & & \\
\hline & Gestational diabetes & Gestational diabetes yes/no & & $2517(3147)^{\star \star}$ \\
\hline \multirow[t]{3}{*}{ Neonatal health (SO) } & \multirow[t]{2}{*}{ Growth measures at birth } & $\begin{array}{l}\text { Birth weight, length and head } \\
\text { circumference }\end{array}$ & \multirow[t]{3}{*}{ Data retrieved from MBRN. } & - \\
\hline & & LGA/SGA & & $1044(1305)^{\star \star}$ \\
\hline & $\begin{array}{l}\text { Newborn adiposity } \\
\text { Preterm delivery }\end{array}$ & $\begin{array}{l}\text { Ponderal index }(\mathrm{kg} / \mathrm{cm} \ddagger)<37 \\
\text { weeks of gestation }\end{array}$ & & $461(577)^{\star \star}$ \\
\hline
\end{tabular}

${ }^{*}$ Numbers needed in each group. Calculated with a statistical power of $80 \%$ and type 1 error of $5 \%$.

†Accounted for a $25 \%$ dropout rate in line with other interventions. ${ }^{30}$

$\ddagger$ Measured at baseline, post intervention and follow-up.

§Healthy Eating Index from The Norwegian Mother, Father and Child cohort (MoBa) (mean score 49.8 \pm 7.3 (SD)) used as a proxy for the planned PRECDIET score. As even small improvements in diet are relevant to public health, we assume an increase of 2 points to have a public health impact. १Estimation based on the $1 \mathrm{~kg}$ decrease in GWG with SD from. ${ }^{28}$

${ }^{* *}$ Based on proportions from Medical Birth Registry of Norway (MBRN), assuming improvement in line with previous group differences in pregnancy. ${ }^{30}$ LGA, large for gestational age; SGA, small for gestational age. 
that it is difficult to get participants to fill in 224 hour recalls, we will also randomise those who have only filled in one 24 hours recall after two reminders of filling in the second. Block randomisation with a 1:1 allocation ratio of eligible participants from a computer-generated list will be used. A statistician (M LeBlanc) with no other role in the data collection has generated the allocation sequence. An email will be sent automatically, informing the participant about which group he/she has been allocated to. In line with the nature of the intervention, this is an open-label study where participants are aware of their intervention status, but the statistician and all involved researchers only have access to anonymised datasets. The automatic recruitment system, which is a separate system from data collection and the intervention, is set up so that only one researcher (ELV) have access can see the email addresses of those who accept to participate. This is not connected to the entry of any data; however, one can see the e-mail-address and the group the person has been automatically drawn to. The list can only be accessed by two persons, one developer and ELV. Blinding of data will only be reversed in the case that a participant withdraws his/her consent for participation. In such cases, data withdrawal will be performed by a project worker and still be anonymised for the researchers performing the analysis.

Following randomisation (see figure 1), participants randomised to the intervention group will be given access to a digital intervention for 26 weeks counted from the day of first access. The control group will receive an email informing about group allocation, highlighting the importance of control groups in research and the value of their continued responses to questionnaires. In addition, as an incentive to participate, all participants finishing the baseline and two first follow-ups will be included in a lottery of $10 \times 5000$ Norwegian Kroner (NOK) gift cards.

Follow-up assessments by questionnaires will be done 6 and 12 months after the intervention. Yearly assessments will be performed until the first child is born, or a maximum of 20 years. If a participant indicates that he/ she is becoming a father/mother, we will ask for consent to use data from the Norwegian birth registry from both parents, regardless of whether the partner has participated actively in the project or not. After the birth of the first child, a final questionnaire will be sent by email to the participating parent.

We will strive to promote participant retention in the study by sending promotional reminders. We will communicate to all participants that continued participation is important for maintaining good retention rates. We will make short, animated films to motivate this. These films will not be sent to the pilot participants.

\section{Pilot study}

We are piloting the study in August-November 2021. The aim of the pilot study is to trial recruitment strategy, assess compliance and retention, testing our data collection instruments, evaluate the automated data-sampling flow and help develop data management and entry processes.
We will further assess at what stages participants drop out and speculate on possible reasons for this and identify whether there are questions that are not responded to. We will use these data to plan strategies for how to reduce dropout, how to handle questionnaires with few entries and more. Further, we will explore group differences between those participating and not regarding gender, age and where they live. We have sent postal invitations to 1000 individuals. The pilot sample is drawn by Statistics Norway to be nationally representative of the population relevant for this study. Preliminary results show that this is not a preferred recruitment method as (1) several addresses are no longer correct and the letters are returned $(n=140)$ and (2) the number of people who have agreed to participate is low (no reminders have been sent). We will analyse the pilot data using the same statistical methods as in the main study. We will not include data from the pilot in the main study.

\section{Development and description of the dietary intervention}

A digital dietary intervention, aiming at promoting the importance and usefulness of a healthy diet for health now, later in life and in the next generation has been developed. The intervention is designed to target both men and women, with a special focus on reaching men due to the less communicated relevance of male preconception diet for the health of prospective children. To aid the intervention development, we conducted short interviews with 34 men and women in the target group about what content they would like from a digital resource aiming to improve diet and what they perceived would motivate them to modify dietary habits (in manuscript). Based on findings from these interviews, we decided to apply self-determination theory to guide the development of promotional messages, aiming to meet participants' need for autonomy, competence and relatedness through the intervention content. ${ }^{18} 19$

The Determinants of Nutrition and Eating framework was used to decide on determinants to be targeted through the intervention. ${ }^{20}$ We targeted modifiable determinants at the individual level with relatively high population-level effect, being Self-regulation, Food Knowledge, Skills and Abilities, Nutrition Knowledge, Health Cognition, Food Habits, Food Beliefs and Eating Regulation.

The resulting digital resource is designed as a webpage with informational videos and texts highlighting the dividends of healthy eating, illustrated recipes and practical information related to meal planning and healthy cooking. All content is in line with official Norwegian dietary recommendations and highlights the importance of food choice for health and well-being. The webpage is designed for weekly advance to a new level with additional information material and recipes to be accessed. Intervention participants will be approached weekly by email, including a short motivational message aimed at inspiring them to visit the website to access new educational content. No other strategies to improve adherence will be used. The duration of the digital intervention is 6 
months and is partly due to (1) costs and timeframe, ${ }^{21}$ (2) stakeholders asking for short messages over some time instead of long messages over a short period and (3) the general perception that less than 6 months is too short to see long-term changes for public health interventions. We will monitor the number of times participants visit the different content on the website and time spent there.

As PREPARED is a low-intensity intervention aiming to promote healthy dietary behaviour and for the general public harm seems unlikely. For vulnerable groups, such as persons with disordered eating could have unintended effects. However, the intervention focuses on the fundamental need for food to thrive and there is no focus on energy balance. Therefore we have not included criteria for discontinuing the intervention, other than at participant request. Further, we will not assess nor prohibit participant participation in other interventions during the intervention period.

\section{Control group}

The control group will not be given access to the intervention website and will not receive any other form of intervention. Neither the intervention group nor the control group will receive feedback regarding individual results.

\section{Outcome measurements}

Primary and secondary outcomes are presented in table 1 including suggestive power calculations for each outcome.

\section{Primary outcomes}

The primary outcome of the trial is the postintervention preconception diet (overall diet and diet quality). Diet quality is assessed by the degree of adherence to Norwegian nutrition and food-based guidelines, indicating a healthier diet. Dietary intake will be assessed using two different tools. At baseline, at the end of the intervention (after 6 months), and 6 months after the intervention is completed, participants will be asked to complete $2 \times 24$-hour dietary recalls using the validated instrument myfood 24. ${ }^{22} 23$ This will yield data on nutrient and food intake (both diet quality measures). In addition, a questionnaire comprising a short newly validated (not yet published) food propensity questionnaire will be used to assess the intake frequency of selected food items relevant to evaluate diet quality by adherence to food-based guidelines at all time points (once a year until the first child is born). This food propensity questionnaire includes frequency questions (times/day) on 33 food items (bread, cereal, spread, dinner foods, sweets and sugary foods and drinks). Nine response alternatives are given, ranging from six times a day or more to never. The questionnaire is designed to capture 6 of the 12 Norwegian dietary guidelines. A score will be developed to quantify adherence to the dietary guidelines.

\section{Secondary outcomes}

The secondary outcomes are health-related quality of life measured by the satisfaction with life scale ${ }^{24}$ and RAND$12 .^{25}$ Maternal pregnancy health (gestational weight gain, preeclampsia, gestational diabetes) and neonatal health (birth weight, birth length, head circumference, gestational age-adjusted weight/length, newborn adiposity and preterm delivery). Maternal pregnancy health and neonatal health are retrieved from the Medical Birth Registry of Norway (MBRN) (see table 1).

\section{Other study parameters}

Several variables will be assessed as potential confounders and effect modifiers. The presented variables in this section are all self-reported. Gender (man/woman/ other), ethnicity (your native tongue, your parents' native tongue), socio-economic status (SES) (economy (house owner, manages unexpected bills), education, work status), area of residence (number of people in city/county), family structure, sleep, physical activity/ sedentary behaviour/screen time (how often and duration), use of tobacco products, having been breastfed as a child, birth weight of the participant (categorical), current height and weight, quality of life (two different scales (see table 1)), Developmental Origins of Health and Disease-knowledge (how they agree on statements on the importance of diet during pregnancy and link to obesity and growth) and whether they are currently trying to conceive. ${ }^{26}$ The questions on birth weight and being breastfed are exploratively used. As we have not included these questions before, response is optional. Our impression is that this is information many knows.

\section{Process evaluation}

We will perform a process evaluation in line with the Medical Research Council (MRC) guidance of 'Process evaluation of Complex interventions, ${ }^{27}$ and will assess implementation, context and mechanisms of impact. Regarding implementation, we will assess fidelity (whether the intervention was delivered as intended, measured by use of the intervention web-site (routine monitoring data, whether they enter the web-page when receiving an e-mail, whether they watch all content or just some of it)), dose (the quantity of intervention implemented (routine monitoring data, for how long are they staying at the intervention page, how often do they enter on a group level)) and 'reach' of intervention (whether the intended audience encounters the intervention, and whether we reach both genders and all age groups). We will explore mechanisms of potential change using quantitative (outcome assessment) and qualitative methods (interviews with users). To evaluate the influence of contextual factors on intervention effects we consider quantitative background variables such as socioeconomic factors, participant being in a relationship or not, and whether actively planning parenthood or not.

\section{Sample size calculation}

Sample-size estimations for selected outcomes are presented in table 1 (25\% dropout included). For our primary outcome, preconception diet, we will assess changes in diet in male and female participants 
separately, necessitating the recruitment of 528 people of both genders. For quality of life (QoL) outcomes, we will need 916 of both genders. Sample size calculation was carried out for most secondary outcomes on pregnancy and child health outcomes (see table 1). The highest number needed to identify a possible effect of the intervention on these outcomes is 2517 in each group, necessitating a total of 5034 participants, equalising 6294 with a $25 \%$ dropout rate. Because of the long follow-up, and uncertainty regarding what time (if) participants get pregnant, we will recruit as many as possible, aiming for at least 7000 participants, hopefully leaving us with the needed numbers in the final analyses. There are several assumptions behind the sample size calculation. First, there is no clear definition of the needed size of a dietary change relevant for public health, so those are decisions based on collective knowledge and literature. Second, a dropout rate of $25 \%$ is somewhat modest, however in line with some of our previous interventions. ${ }^{1728}$ It is, however, important to mention that by linking data to the MBRN, the drop-out will be minimal for secondary outcomes from MBRN, as most will respond to the baseline questionnaire, and intention-to-treat analyses do not rely on follow-up measures of diet for these outcomes. Third, we do not know the effect of the long-term follow-up period on attrition, as few studies have done this before. We, therefore, aim to recruit at least 7000 for baseline.

\section{Statistical analysis}

Analyses will be carried out on an intention to treat basis. Descriptive statistics for baseline characteristics and diet in the two arms will be presented according to sex. Attrition in the two arms will be presented and compared, also stratified by sex.

Measures of diet 6 and 12 months after randomisation (post intervention and 6 months post intervention) are the primary outcome of the study and will be captured by a score type variable; other outcomes include continuous (eg, birth weight) and binary variables (eg, preeclampsia). All will be analysed with an appropriate general linear model, the selection of which will be aided using model diagnostics. Change score analysis that determines the group effect based on the difference between the baseline and the post-treatment score will likely be used for the main outcome-this improves power when the correlation between baseline and follow-up is high as expected for diet outcomes ${ }^{29}$ but we will adjust for baseline if there are unexpected baseline differences in the outcomes. While a statistically principled approach will be used to develop the finer details of the analytical approach, sensitivity analyses will be performed to test the robustness of findings to such decisions.

Per-protocol analyses (predetermined minimum use or fidelity of the digital resource) may also be carried out, but only to get an indication of the power of the intervention components. We will assess formal statistical interactions (eg, sex, age, education and pregnancy intention).
Findings from such subgroup analyses will be used to generate potential new hypotheses that need to be tested.

\section{Data management}

A detailed plan for data entry, coding security and storage has been developed. In short, all person-sensitive data will be stored at the Services for sensitive data (TSD), University of Oslo, a secured server. See figure 2 for an overview of the data storage and handling. When participants register for participation, they will use a two-factor authentication system to enter their data in TSD. All data combined with a person identifying variable will be stored in TSD. Data from myfood24 will be stored at servers at DigitalOcean in Amsterdam temporarily and then stored at TSD, with a secure backup in London (The General Data Protection Regulation (GDPR) compliant). Participants' e-mail addresses and secondary key codes will be collected from TSD and saved on a cloud storage application at the university with a two-factor authentication log-in to recruit for dietary assessment, also in line with GDPR. Further, all data will be stored at TSD. Data will be checked for double registrations routinely, and all variables will be checked for validity/plausibility. Selfreported data entry fields have a restricted range of legal values to minimise errors and outliers, that is, height and weight.

\section{Data monitoring}

We have not included a data monitoring committee in this project. We address the general public (not patients) with messages aimed at motivating dietary change and for the general public harm seems unlikely. The resulting dietary changes are expected to be small, although potentially important. As we believe harm is unlikely (see above), a data monitoring committee was not considered needed. An interim analysis will therefore not be done only for the sake of checking how the intervention is working. We will start evaluating our main hypothesis and combine data from the MBRN when the number of pregnancies in the cohort reaches 1100 . We expect this to take 3-5 years, depending on the age distribution in the recruited sample. No external auditing group will be established.

\section{Participant and public involvement}

We have involved the target group at several stages in the development of the study. First, we had initial discussions with six students in the relevant age group regarding how to communicate sensitive issues how your diet today may affect your children later. This helped us with the framing of the project of not giving advice, merely focusing on information and how the system around food works at different levels. To gain insight into target group opinion and priorities as we were exploring topics for the intervention, we carried out brief interviews in three public places in January 2020. A total of 34 young adults provided informed consent to be interviewed and responded to questions related to motivation for healthy eating, what they needed from a digital intervention to be inspired, 


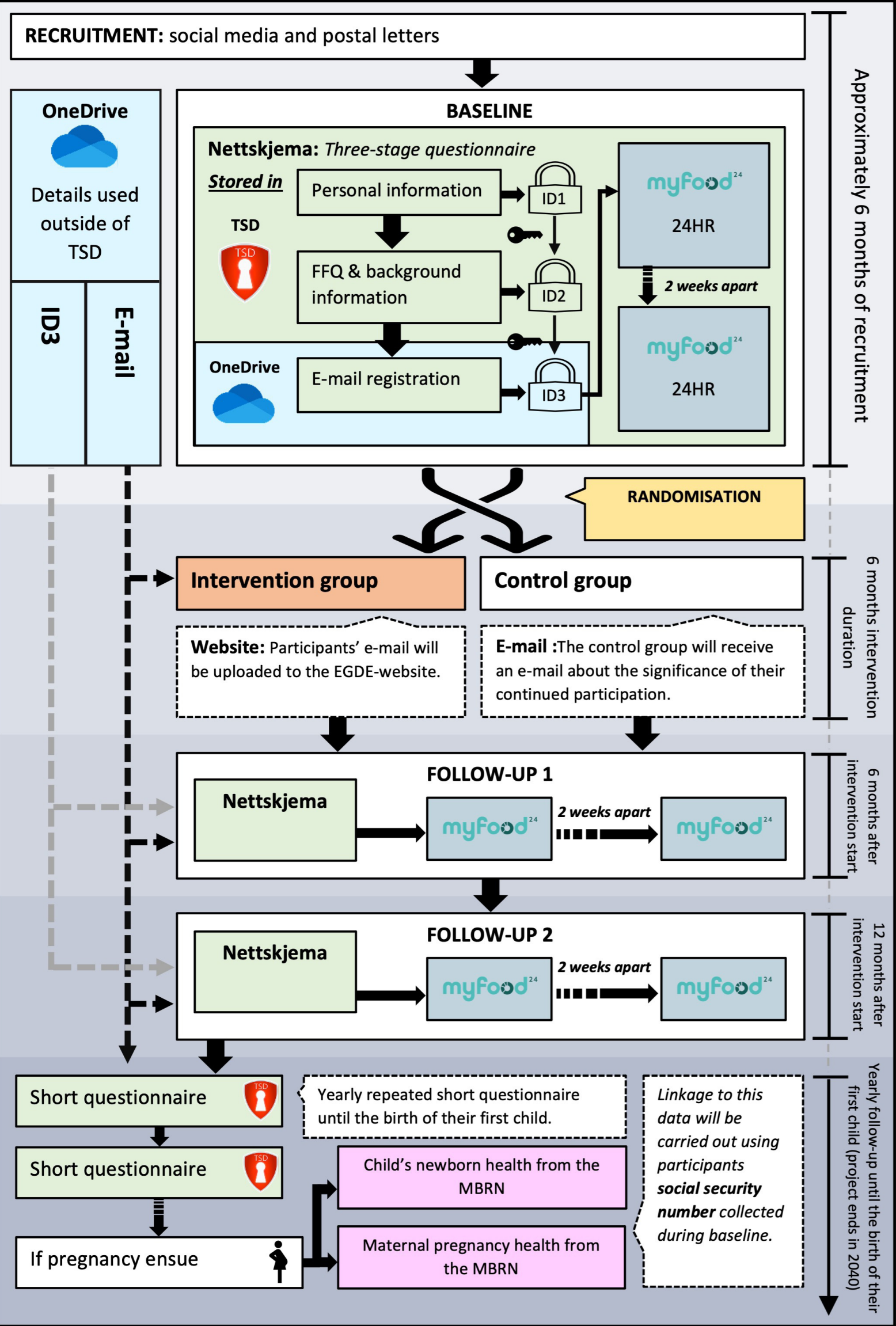

Figure 2 Timeline including data on data safety and storage. MBRN, Medical Birth Registry of Norway; TSD, services for sensitive data. 
whether they believed preconception diet affected future children, and what they thought would motivate the target population to participate in our project. We also carried out 13 focus group discussions where 57 university students consented to participate, addressing preconception diet and how courses or interventions might be carried out to improve diet in these phases.

Further, we involved representatives of the target group in overseeing texts and videos to discover elements or passages that could seem unclear and adapted content accordingly.

The research questions and outcome measures and recruitment strategy were partly informed by information gained in the target group interviews and the focus group discussions, however mainly from the literature pointing to the importance of nutrition and diet in the preconception phase. We will involve the target group when results are to be communicated to participants and the public to secure communication that is non-condemning/ blaming/hurting and just informative.

\section{ETHICS AND DISSEMINATION}

The project has been approved by the Regional Ethics Committee (REC) and our Faculty Ethical Committee (FEC) and the Norwegian Data Protection Service (NSD) (id-numbers: REC: 78104, FEC 20/10119. NSD: 907212). Required protocol modifications will be submitted to the REC the NSD and the trial registry ISRCTN. Participation is voluntary, and participants must first choose to participate on the website before they can provide identification, then digitally actively consent to participation and then start completing the questionnaire. Participants can withdraw their consent without giving any reason. The participants consent to the use of the data in ancillary studies.

As this is an intervention aiming to promote healthy dietary behaviour, harm seems unlikely for the general population. For vulnerable groups, such as persons with disordered eating the intervention could have unintended effects. However, the intervention focuses on the fundamental need for food to thrive and there is no focus on energy balance.

One of the secondary aims of PREPARED is to evaluate the long-term effect of the dietary intervention on maternal and child outcomes. We will collect new consent from both parents for their preconception data to be merged with pregnancy and birth data in the MBRN.

Personal information will be stored in TSD and no personal data will be shared from this project. Only anonymised data will be shared.

For the general public and our participants, we plan to communicate results through a project website and our research group's Twitter account. For healthcare professionals and the research society, we will publish our results in peer-reviewed journals and conferences.

Data sharing plan: We plan to share anonymised data in the UiA deposit Dataverse. This will be done no later than the acceptance for publication of the main findings from the final dataset. We will retain our data for 5 years after data collection has stopped (meaning that data from our baseline will be available no later than 2027 or on publication of main findings). Standard meta-information about the data will be uploaded.

\section{DISCUSSION}

The PREPARED study is one of the first to investigate the potential effects of a dietary intervention during preconception years on participant year-by-year diet and maternal and child outcomes in a future pregnancy. This study has a public health perspective targeting both men and women aged 20-35 in general in line with definitions from Stephenson et al. ${ }^{1}$ Most studies to date have targeted persons planning a pregnancy or specific vulnerable groups. The PREPARED study is planned as an intervention that, if feasible and successful, can be easily scaled up as a public health initiative at a national level and be implemented in other settings. The digital and low cost approach is an advantage for scaling up and makes it easy to include new elements if deemed necessary in potential updated versions.

One strength of this study is the possibility of combining repeated diet information with maternal and child data from the birth registry for participants' future children.

The long duration of the project is one of its strengths, but it also involves methodological challenges regarding loss to follow-up. There is a risk of loss to follow-up in several phases; therefore, there is a need to build a sense of identity to the study to make the participants continue responding to a short questionnaire every year. We will make the follow-up questionnaires less time-consuming (5-10 min). In addition, our choice of dietary methods, $2 \times 24$-hour dietary recalls in combination with a short dietary screener provide less systematic errors and is no more time consuming than an Food frequency questionnaire (FFQ), but the fact that one has to respond twice at 2 week intervals gives a risk of loss to follow-up. We therefore want to randomise those with only one dietary recall if they have not filled in the second after two reminders.

The age range that we target may be a limitation of our study, since it is very broad and include participants in different phases of life. The age range was decided on using two principles: (1) broad reach and (2) 'do not harm' addressing these issues. With the broad approach, we first wanted to include 18-45 year-olds as this roughly corresponds to fertile age above the age of majority. ${ }^{18}$ However most 18-19year-olds still attend public schools and live at home, and as we wanted to approach the young adults themselves (not their parents), we chose the lower age limit to be 20 years. We discussed the upper age limit with several persons from our target population and concluded that being approached with a study focusing on your first child might seem strange or difficult if you do not yet have, and truly want, children. We, therefore, chose 20-35 years of age as the most appropriate age 
group to target. Still, this age span includes participants in potentially very different phases of life. Emerging adulthood has recently been proposed as a new life stage between adolescence and young adulthood, lasting from ages about $18-25$. This period is characterised by identity exploration, trying out various life experiences and not yet taking the responsibility as is normative in adulthood. We also include ages 25-35, where more take on adult responsibility. The average parental age of the first child is around 32 in Norway, this period is, therefore, crucial to include. Another limitation is the intervention period of 6 months, which may be a bit short to achieve lasting changes. This might also have been approached more thoroughly by early phase trials, as suggested by Voils et $a l .{ }^{21}$ However, we believe that this is new knowledge for young adults and can therefore motivate change even if it is only provided for 6 months.

This study is one of the first to evaluate the potential effects of a dietary intervention targeting adults aged 20-35 without biological children. We will evaluate the effect of the intervention on aspects of diet, and the longterm effect on newborn health of their future children. The long follow-up time and the large sample size also make this study an interesting and important cohort. Findings regarding potential intervention effect on adult diet will be reported in 2-3 years, while preliminary effects on child health are expected in 5-10 years. This study will, if successful, provide an easily scalable intervention to approach the general young adult population to promote a healthy start for their future children. In addition, the study will provide unique long-term data on young adults' diet in preconception years.

\section{AUTHORSHIP ELIGIBILITY GUIDELINES}

All included authors of the protocol paper will be invited to contribute as coauthors for the first result paper. For future papers, Principal Investigator (PI) and co-PI will be asked to be included and will adhere to author guidelines, others will be asked on request and must qualify for authorship by participating in analyzing, writing or interpretation.

\section{Twitter Nina Cecilie Øverby @0verbyNina}

Acknowledgements We thank the University of Agder for providing funding for this project and we thank Marissa LeBlanc for providing the randomization list, and our research assistant Camilla Bjornes for helping with project meetings minutes and helping to prepare the questionnaires.

Contributors NCO, ERH and FNV conceived the main conceptual ideas and applied for funding. FNV led the work on planning the recruitment strategy, assisted by ELV. ERH led the work on the development of the intervention, assisted by ELV, NCO, ACM and LS. ACM led the work on the choice of methods, developing questionnaires, assisted by ELV, LS, NCO. NCO led the work on developing the flow and system of the project (see figure 2) and the data management, assisted by ELV, LS, DE, ACM. NCO is the PI in collaboration with ERH as co-PI. AKW has been advising on the choice of statistical approaches and study design. NCO drafted the protocol paper, and all authors have critically reviewed, commented and revised it. All authors have approved the last version of the paper.

Funding No trial sponsors outside the University of Agder. Award number: 1325 Lifecourse Nutrition, UiA. The University of Agder had no role in study design, data collection, study management, data analysis, or interpretation of data, nor any role in writing the protocol or deciding where to submit the report for publication.

Competing interests None declared.

Patient and public involvement Patients and/or the public were involved in the design, or conduct, or reporting, or dissemination plans of this research. Refer to the Methods section for further details.

Patient consent for publication Not applicable.

Provenance and peer review Not commissioned; externally peer reviewed.

Open access This is an open access article distributed in accordance with the Creative Commons Attribution Non Commercial (CC BY-NC 4.0) license, which permits others to distribute, remix, adapt, build upon this work non-commercially, and license their derivative works on different terms, provided the original work is properly cited, appropriate credit is given, any changes made indicated, and the use is non-commercial. See: http://creativecommons.org/licenses/by-nc/4.0/.

ORCID iD

Nina Cecilie Øverby http://orcid.org/0000-0002-1871-041X

\section{REFERENCES}

1 Stephenson J, Heslehurst N, Hall J, et al. Before the beginning: nutrition and lifestyle in the preconception period and its importance for future health. Lancet 2018;391:1830-41.

2 Eberle C, Kirchner MF, Herden R, et al. Paternal metabolic and cardiovascular programming of their offspring: a systematic scoping review. PLoS One 2020;15:e0244826.

3 Fleming TP, Watkins AJ, Velazquez MA, et al. Origins of lifetime health around the time of conception: causes and consequences. Lancet 2018;391:1842-52.

4 Barker M, Dombrowski SU, Colbourn T, et al. Intervention strategies to improve nutrition and health behaviours before conception. Lancet 2018;391:1853-64.

5 Hieronimus B, Ensenauer R. Influence of maternal and paternal preconception overweight/obesity on offspring outcomes and strategies for prevention. Eur J Clin Nutr 2021 doi:10.1038/s41430-021-009207

6 Patton GC, Olsson CA, Skirbekk V, et al. Adolescence and the next generation. Nature 2018;554:458-66.

7 Nga HT, Quyen PN, Chaffee BW, et al. Effect of a nutrient-rich, foodbased supplement given to rural Vietnamese mothers prior to and/or during pregnancy on birth outcomes: a randomized controlled trial. PLoS One 2020;15:e0232197.

8 Steegers-Theunissen R, Hoek A, Groen H, et al. Pre-conception interventions for subfertile couples undergoing assisted reproductive technology treatment: modeling analysis. JMIR Mhealth Uhealth 2020;8:e19570.

9 Gardiner P, Bickmore T, Yinusa-Nyahkoon L, et al. Using health information technology to engage African American women on nutrition and supplement use during the preconception period. Front Endocrinol 2020;11:571705.

10 Kumaran K, Krishnaveni GV, Suryanarayana KG, et al. Protocol for a cluster randomised trial evaluating a multifaceted intervention starting preconceptionally-Early interventions to support trajectories for healthy life in India (Einstein): a healthy life trajectories initiative (HeLTI) study. BMJ Open 2021;11:e045862.

11 Hanson MA, Bardsley A, De-Regil LM, et al. The International Federation of Gynecology and Obstetrics (FIGO) recommendations on adolescent, preconception, and maternal nutrition: "Think Nutrition First". Int J Gynaecol Obstet 2015;131:S213-53.

12 GBD 2017 Risk Factor Collaborators. Global, regional, and national comparative risk assessment of 84 behavioural, environmental and occupational, and metabolic risks or clusters of risks for 195 countries and territories, 1990-2017: a systematic analysis for the global burden of disease study 2017. Lancet 2018;392:1923-94.

13 Totland TH, Melnæs BK, Lundeberg-Hallen N. Norkost 3 a national dietary survey among men and women in Norway aged 18-70 years, 2010-11 (in Norwegian). Oslo, 2012.

14 Valen EL, Engeset D, Øverby NC, et al. StudentKost: a crosssectional study assessing college students' diets: reason for concern? J Nutr Sci 2020;9:e39.

15 Kandel P, Lim S, Pirotta S, et al. Enablers and barriers to women's lifestyle behavior change during the preconception period: a systematic review. Obes Rev 2021;22:e13235.

16 SPIRIT 2013 checklist. Available: http://www.spirit-statement.org/ wp-content/uploads/2013/01/SPIRIT-Checklist-download-8Jan13. pdf [Accessed 30 Jun 2021]. 
17 Helle C, Hillesund ER, Wills AK, et al. Evaluation of an eHealth intervention aiming to promote healthy food habits from infancy -the Norwegian randomized controlled trial early food for future health. Int $J$ Behav Nutr Phys Act 2019;16:1.

18 Deci EL, Ryan RM, Theory S-D. In Handbook of theories of social psychology. London: SAGE Publications Ltd, 2012.

19 Edmunds J, Ntoumanis N, Duda JL. Testing a self-determination theory-based teaching style intervention in the exercise domain. Europ J Soc Psychol 2007;38.

20 Stok FM, Hoffmann S, Volkert D, et al. The DONE framework: creation, evaluation, and updating of an interdisciplinary, dynamic framework 2.0 of determinants of nutrition and eating. PLoS One 2017;12:e0171077.

21 Voils Cl, King HA, Maciejewski ML, et al. Approaches for informing optimal dose of behavioral interventions. Ann Behav Med 2014;48:392-401.

22 Wark PA, Hardie LJ, Frost GS, et al. Validity of an online 24-h recall tool (myfood24) for dietary assessment in population studies: comparison with biomarkers and standard interviews. BMC Med 2018;16:136.

23 Salvesen L, Engeset D, Øverby NC, et al. Development and evaluation of image-series for portion size estimation in dietary assessment among adults. J Nutr Sci 2021;10:e3.
24 Diener E, Emmons RA, Larsen RJ, et al. The satisfaction with life scale. J Pers Assess 1985;49:71-5.

25 Rand Health Care. 12-Item short form survey (SF-12). Available: https://www.rand.org/health-care/surveys_tools/mos/12-item-shortform.html [Accessed 30 Jun 2021].

26 McKerracher L, Moffat T, Barker M, et al. Knowledge about the developmental origins of health and disease is independently associated with variation in diet quality during pregnancy. Matern Child Nutr 2020;16:e12891.

27 Moore GF, Audrey S, Barker M, et al. Process evaluation of complex interventions: Medical Research Council guidance. $B M J$ 2015;350:h1258.

28 Sagedal LR, Øverby NC, Bere E, et al. Lifestyle intervention to limit gestational weight gain: the Norwegian fit for delivery randomised controlled trial. BJOG 2017;124:97-109.

29 Vickers AJ, Altman DG. Statistics notes: analysing controlled trials with baseline and follow up measurements. BMJ 2001;323:1123-4.

30 Wu T, Gao X, Chen M, et al. Long-term effectiveness of diet-plusexercise interventions vs. diet-only interventions for weight loss: a meta-analysis. Obes Rev 2009;10:313-23. 